\title{
Indicaciones endoscópicas en el paciente con trasplante hepático
}

\author{
Graciela Elia Castro-Narro ${ }^{1 *}$ y Yahvé Iván López-Méndez ${ }^{2}$ \\ ${ }^{1}$ Departamento de Gastroenterologia, Instituto Nacional de Ciencias Médicas y Nutrición "Salvador Zubirán"; 2 Departamento de Gastroentrología, \\ Endoscopía y Ecografía Endoscópica. Hospital Médica Sur, Ciudad de México, México
}

\begin{abstract}
Resumen
El trasplante hepático ortotópico (THO) es el tratamiento estándar para la enfermedad hepática terminal. Las complicaciones biliares siguen siendo la principal causa de morbilidad, pérdida del injerto y mortalidad después de trasplante. Las complicaciones biliares post-trasplante incluyen: estenosis anastomóticas, estenosis no anastomóticas, fuga biliar y coledocolitiasis. Las estenosis y fugas biliares son las complicaciones más frecuentes post-trasplante, generalmente se ven como complicaciones tardías, la mediana de tiempo de presentación es de 5.9 meses. La incidencia reportada es del 5-15\% después del trasplante hepático del donante cadavérico y del 20-34\% después del trasplante de donador vivo seguidos por litos biliares, detritos, biloma y hemobilia'. Las opciones de tratamiento incluyen técnicas endoscópicas, intervenciones percutáneas transhepáticas y procedimientos quirúrgicos. La colangiopancreatografía retrógrada endoscópica (CPRE) es la herramienta terapéutica de elección para el tratamiento de estenosis, coledocolitiasis y fugas biliares por ser menos invasiva, más segura y efectiva; las tasas de complicaciones de la CPRE son de 5-9\%, similares a las de CPRE sin trasplante. La terapia percutánea se considera segunda línea y la opción quirúrgica está reservada como terapia de rescate cuando todas las modalidades anteriores han fracasado².
\end{abstract}

Palabras clave: Trasplante hepático ortotópico. Estenosis biliares. Fugas biliares. Colangiopancreatografía retrógrada endoscópica.

\section{Estenosis biliares post-tho}

Las estenosis biliares post-trasplante hepático se clasifican como anastomóticas (EA) y no anastomóticas (ENA). Las EA son únicas, más cortas y localizadas en el sitio anastomótico, Las ENA suelen ser más largas, múltiples, difusas y más proximales (cerca del hilio y los conductos biliares intrahepáticos).

\section{Estenosis anastomótica}

La identificación temprana de la estenosis se correlaciona con una mejor respuesta a la colocación de la endoprótesis a corto plazo (3-6 meses). Se ha demostrado que la EA dentro de los 3 meses posteriores al trasplante tienen mejor pronóstico. El pilar del manejo de la estenosis anastomótica gira en torno a la terapia con CPRE. La mayoría de los pacientes requerirán múltiples sesiones de CPRE cada 3 meses, con colocación de la endoprótesis y dilatación durante 1 a 2 años.

El tratamiento endoscópico estándar consiste en CPRE con esfinterotomía más colocación de endoprótesis con o sin dilatación neumática progresiva. La tasa de éxito es de $80-90 \%$. Si una estenosis no responde

\section{Correspondencia:}


a la terapia endoscópica o percutánea, la cirugía puede estar indicada.

La endoprótesis plástica tiene dos limitaciones principales: con frecuencia se requiere múltiples endoprótesis para tratar las estenosis anastomóticas, y como consecuencia de su tendencia a obstruirse dentro de los siguientes tres meses, deben reemplazarse. Además, la repetición de CPRE tiene riesgos como colangitis, pancreatitis y perforación, con una tasa de complicación de 2-6\%. Un estudio en el que se realizó dilatación con balón seguida de la colocación de múltiples endoprótesis de máximo $10 \mathrm{Fr}$, con repeticiones de CPRE cada 3 meses hasta la resolución completa de la estenosis por fluoroscopia mostró una tasa de éxito de 80 a 95\%, con una recurrencia de 20 a $35 \%{ }^{3}$.

Las prótesis metálicas autoexpandibles (PMAE) pueden tener varias ventajas. En primer lugar, la colocación es fácil y rápida, con tasas de éxito técnico entre el 86 y $100 \%$. Un estudio aleatorizado con 32 pacientes reportó éxito de $100 \%$ en pacientes con PMAE contra $80 \%$ con endoprótesis plásticas. Otro estudio con 16 pacientes informó una tasa de éxito del $87.5 \%$ a los 10 meses de seguimiento, aunque hubo una alta tasa de migración de la endoprótesis $(37.5 \%)^{4}$.

\section{Estenosis no anastomóticas}

Se ha demostrado que el tiempo prolongado de isquemia fría es responsable del desarrollo de la ENA. Las ENA son más difíciles de manejar que las EA, ya que el tratamiento en cada caso debe ser individualizado. Por lo tanto, es difícil hacer recomendaciones generalizadas para el tratamiento de la ENA. El éxito de dilatación con balón y colocación de stent plástico es de $50-70 \%$. La dilatación repetida con colocación de endoprótesis parece ser la forma de tratamiento más aceptada. El éxito del tratamiento depende de la gravedad de la estenosis, el número y la ubicación. Las estenosis extrahepáticas generalmente responden mejor a la terapia ${ }^{5}$.

\section{Fuga biliar}

Las fugas biliares tempranas ( $<4$ semanas) generalmente ocurren debido a problemas técnicos relacionados con la cirugía, incluyendo: sangrado activo en el extremo del conducto biliar, disección excesiva del tejido periductal en el momento de la obtención, tensión en la anastomosis ductal, sutura incorrecta del muñón del conducto cístico o uso de electrocauterio para controlar el sangrado. Las fugas biliares tardías ( $>4$ semanas) suelen estar relacionadas con la extirpación prematura del tubo en T, momento en el que puede haberse desarrollado una fístula. En un estudio, el $31 \%$ de los pacientes con un tubo en T informó una fuga biliar, y el $7 \%$ fue tardía $^{6}$. El tratamiento endoscópico suele ser muy eficaz, el objetivo de la endoterapia es reducir el gradiente de presión transpapilar entre el árbol biliar y el duodeno mediante una endoprótesis con o sin esfinterotomía.

La CPRE junto con esfinterotomía y la colocación de la endoprótesis biliar plástica, se ha convertido en el método preferido para diagnosticar y tratar la fuga biliar con altas tasas de éxito y bajas tasas de complicaciones, con una tasa de éxito del 90-95\%. La endoprótesis generalmente se mantiene en su lugar durante varias semanas para asegurar una adecuada cicatrización de la fuga. Después de un manejo conservador exitoso y en ausencia de cualquier patología, se retira la endoprótesis en 2-3 meses?.

Un estudio reportó una alta tasa de éxito de PMAE del $87.5 \%$ en el tratamiento de las fugas biliares asociadas a la EA en una serie corta de pacientes refractarios a la terapia endoscópica estándar. Sin embargo, hubo una tasa considerable de estenosis secundarias (47\%) después de la eliminación de la endoprótesis, la mayoría de las cuales se manejaron con éxito utilizando una endoprótesis plástica, y se utilizó una segunda PMAE solo en un caso ${ }^{8}$.

\section{Defectos de llenado}

Los litos, lodo y detritos de la vía biliar post-trasplante pueden ocurrir en cualquier momento después del trasplante, con una incidencia reportada de 5 a $10 \%$. Los litios biliares ocurren con una mediana de 19 meses después del trasplante. Estos defectos del llenado se asocian fuertemente con eventos isquémicos y generalmente se acompañan de otras complicaciones, la más común la estenosis.

Teóricamente, cualquier cosa que aumente la viscosidad de la bilis o reduzca el flujo puede predisponer a la formación de lodos y litos ${ }^{9}$. Se cree que el daño de la mucosa del conducto biliar debido a una obstrucción, isquemia o infección desempeña un papel en el desarrollo de los detritos. El síndrome del detrito biliar marca la presencia de detritos marrones múltiples, duros y pigmentados que causan obstrucción. La incidencia reportada es de 2.5 a $18 \%$. Se cree que la patogenia de esta afección es multifactorial: el rechazo celular agudo, la isquemia, la infección y la obstrucción biliar debido a la estasis en pacientes con o sin estenosis del conducto biliar son factores contribuyentes. La terapia endoscópica 
CPRE con esfinterotomía, litotripcia y extracción de litos es exitosa para tratar la mayoría de los defectos de llenado con una eficacia que oscila entre $90-100 \%$, por lo que este procedimiento se considera la herramienta inicial. En pacientes con anatomía no alterada, se puede lograr una esfinterotomía estándar seguida de una extracción de litos y lodo o extracción con canasta sin ninguna limitación importante. En pacientes con una anatomía que ha sido alterada por anastomosis bilioentérica, se puede lograr lo mismo usando versiones enteroscópicas modificadas más largas.

\section{Conflicto de intereses}

No existen conflictos de interés. Sin patrocinio de la industria.

\section{Bibliografía}

1. Wadhawan M, Kumar A, Gupta S, et al. Post-transplant biliary complications: An analysis from a predominantly living donor liver transplant center. J Gastroenterol Hepatol 2013; 28:1056-1060.

2. Shin M, Joh JW. Advances in endoscopic management of biliary complications after living donor liver transplantation: Comprehensive review of the literature. World J Gastroenterol 2016; 22:6173-6191.

3. Costamagna G, Pandolfi M, Mutignani M, et al. Long-term results of endoscopic management of postoperative bile duct strictures with increasing numbers of stents. Gastrointest Endosc 2001; 54:162-168.

4. Traina M, Tarantino I, Barresi L, et al. Efficacy and safety of fully covered self-expandable metallic stents in biliary complications after liver transplantation: a preliminary study. Liver Transpl 2009; 15:1493-1498.

5. Kochhar G., Parungao J.M., Hanouneh I., et. al. Biliary complications following liver transplantation. World J Gastroenterol. 2013; 19; 2841-2846.

6. Thuluvath PJ, Pfau PR, Kimmey MB, Ginsberg GG. Biliary complications after liver transplantation: the role of endoscopy. Endoscopy 2005; 37:857-863.

7. Moy B., Birk J. A Review on the Management of Biliary Complications after Orthotopic Liver Transplantation. J Clin Transl Hepatol. 2019; 7: 61-71.

8. Villa NA, Harrison ME. Management of Biliary Strictures After Liver Transplantation. Gastroenterol Hepatol 2015; 11:316-328.

9. Woo Lee H., Hassan Shah N., Koo Lee S. An Update on Endoscopic Management of Post-Liver Transplant Biliary Complications. Clin Endosc. 2017; 50: 451-463. 